\title{
Minerals in the nutrition of extremely low birth weight infants
}

\author{
Cleide E. P. Trindade*
}

\begin{abstract}
Objective: To review the literature on the role of calcium, phosphorus and trace elements in the nutrition of extremely low birth weight infants, considering their importance for metabolism, bone mineralization and as dietary components.

Sources of data: MEDLINE, the Cochrane Database of Systematic Reviews and books on nutrition were searched between 1994 and 2004. Original research studies and reviews were selected.

Summary of the findings: Extremely preterm infants are frequently growth-restricted at hospital discharge as a consequence of difficulties in the provision of adequate nutrition. The long-term effects of this growth restriction need to be determined. There is a paucity of studies about the role of minerals, especially micronutrients, in the nutrition of extremely preterm infants. The principal focus of this review was on calcium and phosphorus metabolism, bone mineralization and parenteral and enteral supplementation. A critical evaluation of post-discharge nutrition and its influence upon growth and bone mineralization was presented. Selenium and zinc requirements and the role of selenium as an antioxidant with possible effects on free radical diseases of the preterm infant were discussed.

Conclusions: Extremely preterm infants have low mineral reserves and, as a consequence, may have deficiencies in the postnatal period if they do not receive parenteral or enteral supplementation. More studies are needed to elucidate the actual requirements and the appropriate supplementation of micronutrients. There are controversies about the outcome and the influence of post-discharge nutrition on bone disease of prematurity.
\end{abstract}

J Pediatr (Rio J). 2005;81(1 Suppl):S43-S51: Infant, preterm, minerals, calcium, selenium, micronutrients, nutrition.

\section{Introduction}

Extremely preterm or extremely low birthweight preterm infants are those weighing less than $1,000 \mathrm{~g}$. These newborns are extremely immature; therefore, nutrition must be adjusted on a case-by-case basis, and not based only on birthweight. There are no accurate definitions of their nutritional requirements, so their growth and metabolism must be frequently monitored.

Due to the advanced care preterm infants have been provided with, the survival rate of infants with low birthweight and low gestational age has increased. These preterm

* Professor, School of Medicine of Botucatu, Universidade Estadual Paulista (UNESP), Botucatu, SP, Brazil.

Suggested citation: Trindade CE. Minerals in the nutrition of extremely low birth weight infants. J Pediatr (Rio J). 2005;81 (1 Suppl):S43-S51. infants have their own metabolic characteristics and a large number of diseases and complications in the postnatal period, making their nutrition a real challenge. Nowadays, the postnatal nutrition of these newborn infants has risen in importance, since fetal malnutrition, and possibly, postnatal malnutrition have been considered in epidemiological studies to play a crucial role in the determination of adult diseases such as diabetes, hypertension and heart diseases. Therefore, neonatologists have a greater responsibility to provide these newborn infants with a nutrition that ensures appropriate physical growth and neuropsychomotor development. Given this scenario, the nutrition of extremely preterm infants using mineral supplementation has become important, due to the fact that minerals have different functions in the body, although little is known about them, especially with regard to the nutrition of extremely preterm infants. As the role of some minerals has been underinvestigated, it should be underscored that calcium 
and phosphorus are the most widely studied minerals in the literature, and this is so because of acute metabolic disorders in the immediate neonatal period, such as early-onset neonatal hypocalcemia, as well as late-onset manifestations, such as metabolic bone disease of prematurity. However, several minerals such as zinc, magnesium, iron, selenium and other microelements are also important to the nutrition of preterm infants, although most of them do not show evident clinical manifestations in the absence of these elements.

\section{Calcium and phosphorus}

Calcium ( $\mathrm{Ca}$ ) and phosphorus ( $\mathrm{P}$ ) are important to fetal and postnatal bone mineralization. The period of greater skeletal development and bone mineralization occurs in the third trimester of gestation when the fetus incorporates large amounts of calcium and phosphorus, on average 120 to $140 \mathrm{mg} / \mathrm{kg} / \mathrm{per}$ day and 60 to $75 \mathrm{mg} / \mathrm{kg} /$ day, respectively.

Mineralization levels increase exponentially between 24 and 37 weeks of gestation, resulting in $80 \%$ of mineral accumulation in the third trimester. As a result, infants born between 24 and 34 weeks will be deprived of the intrauterine supply of calcium, affecting intrauterine and postnatal bone mineralization.

Estimates of calcium requirements in preterm infants are inferred from the chemical composition of fetal tissues, calculated at several gestational ages. Although these methods have been criticized, it is agreed that mineralization levels range from $120 \mathrm{mg} / \mathrm{kg} / \mathrm{per}$ day at 26 weeks to $130-140 \mathrm{mg} / \mathrm{kg} /$ day at $36^{1}$ weeks and between 150 and $155 \mathrm{mg} / \mathrm{kg} /$ day between 36 and 38 weeks of gestation. Bone mineral content (BMC) measurement of newborn infants of different gestational ages, determined by photon absorption, shows that the mineral content of the distal third of the radius increases from $25 \mathrm{mg} / \mathrm{cm}$ at 26 weeks to $65 \mathrm{mg} / \mathrm{cm}$ at 36 weeks. BMC and bone area (BA) measured by dual-energy x-ray absorptiometry (DEXA) revealed that both measurements are correlated with body weight, length and gestational age. ${ }^{2}$

DEXA is considered the most accurate noninvasive method to define in vivo bone mineralization. By using this technique and calculating $\mathrm{BMC}$ and $\mathrm{BA}$, one observes that both measurements are correlated with body weight, length and gestational age. In a multivariate analysis, body weight was the major predictor of these parameters. ${ }^{2}$

The evaluation of Ca mineralization in the fetal period has practical implications, as in the postnatal period, a newborn infant must receive enough calcium for bone mineralization similar to that obtained in utero. Therefore, in the postnatal period of extremely preterm infants, the aim is to provide nutrition so that these infants can make up for intrauterine growth and mineralization. In order for mineralization to occur, there must be bone collagen synthesis, which requires a high intake of protein and energy from extremely low birthweight infants. Along with skeletal growth, calcium and phosphorus must be provided in appropriate amounts so that mineralization takes place. ${ }^{2}$ In extremely preterm infants, some factors make it difficult to achieve growth and mineralization goals, and that is why metabolic bone disease occurs in approximately $50 \%$ of these newborn infants.

Immediately after birth, calcium supplementation is used to prevent or treat neonatal hypocalcemia, which is frequent in extremely preterm infants and results from the interruption of the placental supply of calcium, from high levels of calcitonin that persist on the first days of life and from the insufficient release of parathyroid hormone, due to the immature response of parathyroid glands. ${ }^{3}$ In the postnatal period, there are some difficulties in providing higher amounts of $\mathrm{Ca}$ and $\mathrm{P}$ because these extremely preterm infants usually have intercurrent events such as respiratory distress syndrome, peri-intraventricular hemorrhage and chronic lung disease. These infants are usually dehydrated and have food intolerance, conditions that reduce the supply of proteins, energy, calcium and phosphorus.

\section{Parenteral calcium supply}

Parenteral nutrition is the major source of feeding for extremely preterm infants on the first days of life, until they can tolerate enteral nutrition and receive appropriate amounts of nutrients, which enable growth. However, it is difficult to provide sufficient amounts of $\mathrm{Ca}$ and $\mathrm{P}$ that meet intrauterine requirements through parenteral nutrition. The solubility of calcium in the presence of phosphorus is a limiting factor, in addition to several factors related to the maintenance of the stability of the solution such as: source of calcium, concentration, solution $\mathrm{pH}$, glucose concentration, order of the components, temperature of the solution.

The most widely used solutions consist of calcium gluconate associated with monobasic or dibasic phosphate salts. Solubility improves when phosphate salts are added before calcium salts and by using higher concentrations of amino acids. New formulations containing glycerophosphate and monobasic phosphates allow providing higher amounts of calcium and phosphorus, up to $86 \mathrm{mg} / \mathrm{dl}$ of calcium and $46 \mathrm{mg} / \mathrm{dl}$ of phosphorus, but these concentrations are yet insufficient for appropriate bone mineralization to occur. ${ }^{2}$

In 1993, Koo \& Tsang 4 observed retention of 88 to $94 \%$ and of 83 to 97 of calcium and phosphorus, respectively, when they evaluated different metabolic balance studies in stable newborn infants and in those on parenteral nutrition receiving 58 to $76 \mathrm{mg} / \mathrm{kg} /$ day of calcium and 38 to $54 \mathrm{mg} / \mathrm{kg} /$ day of phosphorus. They concluded that 60 to $70 \%$ of intrauterine mineralization could be obtained from usual parenteral solutions. An important factor for stability of the solution and greater retention is the $\mathrm{Ca}: \mathrm{P}$ ratio. As to the weight of these elements, the $1.3: 1.0$ to $1.7: 1.0$ ratio has been considered to retain $\mathrm{Ca}$ and $\mathrm{P}$ appropriately. ${ }^{4}$ In 2000 , Rigo et al. ${ }^{2}$ considered that a Ca:P ratio of $1.7: 1.0$ allows for maximum administration and retention of calcium and phosphorus parenterally. 


\section{Enteral supply of calcium and phosphorus}

When Ca and $\mathrm{P}$ are provided through fortified breastmilk or special preterm formulas, we should consider that minerals retained by the body would be the result of the total amount supplied and the total amount absorbed by the intestine and the amount eliminated by the kidney and feces.

Intestinal absorption of calcium occurs by active transport and facilitated diffusion. Active absorption is located in the proximal portion of the small intestine, and is dependent on vitamin $\mathrm{D}$. The average Ca absorption in newborns who receive preterm formula ranges from 36 to $75 \%$ of the ingested amount, similar to the one observed in calciumfortified breastmilk. ${ }^{4}$

The absorption of phosphorus in the small intestine occurs by simple diffusion, facilitated diffusion and by the cotransport of sodium and phosphorus. ${ }^{4}$ In phosphorus absorption, there is interference of 1,25 dihydroxyvitamin $D$ and of the parathyroid hormone. One may see that phosphorus retention is elevated in unsupplemented breastmilk due to the low concentration of $\mathrm{P}$, which amounts to $90 \%$ of the ingested amount, and approximately $50 \%$ when milk has a high content of phosphorus. ${ }^{4}$

Several factors interfere with Ca absorption, besides vitamin D. In newborn infants, vitamin $D$ requirements are influenced by its storage at birth, which depends not only on maternal storage, but which also varies according to the length of pregnancy and to geographical region. ${ }^{2}$ To be absorbed, Ca must be in an ionic form because insoluble fractions are poorly absorbed. It has better solubility in the form of chloride, citrate and carbonate, compared to calcium in the form of phosphate, which should therefore be avoided in formulas. ${ }^{2}$ It also has better solubility in the gluconate-glycerophosphate form. ${ }^{2}$ The amount and quality of fat influence calcium absorption by forming soaps and reducing absorption. In breastmilk, the position of palmitate in carbon 2 of the triglyceride improves calcium bioavailability.

\section{Calcium content in breastmilk and formulas}

Studies on breastmilk revealed that calcium content in the initial colostrum is on average $160 \mathrm{mg} / \mathrm{l}$, increasing to $256 \mathrm{mg} / \mathrm{I}$ on the third day, and maintaining these values up to third month, when it slowly declines to $176 \mathrm{mg} / \mathrm{l}$ around one year. ${ }^{5}$ The content of a preterm infant's mother's breastmilk does not differ from that of a full-term infant's mother's in terms of calcium and phosphorus in the first month of life.

These data indicate that the mineral content of breastmilk is insufficient to keep postnatal mineralization in very low birthweight infants at the levels observed in the intrauterine period. Consequently, breastmilk supplements were developed, including different formulations regarding the contents of calcium, phosphorus, proteins and minerals. Preterm formulas were also developed so as to provide large amounts of calcium and phosphorus with the aim of improving the bone mineralization of these preterm infants.

The composition of these supplements, the formulas and retention of calcium and phosphorus is diversified, which in a final analysis, is the key factor for bone mineralization. Thus, in 1993, Koo \& Tsang, ${ }^{4}$ in research studies described by six authors, evaluated mineral balance in preterm infants fed breastmilk combined with calcium and phosphorus or with commercially available supplements and in those fed preterm milk-based formulas, with different calcium and phosphorus contents, described by seven authors. In 2000, Rigo et al., ${ }^{2}$ after evaluating three different breastmilk supplements and 12 preterm formulas, found a large variation in the composition, absorption and retention of calcium and phosphorus. Some data obtained from these two authors are shown in Table 1.

Table 1 - Mineral balance in preterm newborns receiving enteral nutrition

\begin{tabular}{|c|c|c|c|c|c|c|c|}
\hline \multicolumn{8}{|c|}{ Breast milk and dietary supplements } \\
\hline & \multirow[t]{2}{*}{ Supplement } & \multicolumn{3}{|c|}{ Calcium (mg/kg/day) } & \multicolumn{3}{|c|}{ Phosphorus (mg/kg/day) } \\
\hline & & Intake & Absorption & Retention & Intake & Absorption & Retention \\
\hline Salle et al. ${ }^{6}$ & $\mathrm{Ca}+\mathrm{P}$ & 90 & 66 & 63 & 62 & 58 & 53 \\
\hline Ehrenkranz et al. ${ }^{7}$ & $\begin{array}{l}\text { Cow's milk-based } \\
\text { formula }\end{array}$ & 123 & 92 & 82 & 72 & 61 & 58 \\
\hline Schanler et al. ${ }^{8}$ & $\begin{array}{l}\text { Cow's milk-based } \\
\text { formula }\end{array}$ & 137 & 48 & 43 & 71 & 63 & 39 \\
\hline \multicolumn{8}{|c|}{ Preterm formula } \\
\hline Rigo et al. ${ }^{9}$ & PT $F_{1}$ & $91 \pm 14$ & $51 \pm 17$ & $45 \pm 18$ & $63 \pm 9$ & $89 \pm 6$ & $42 \pm 8$ \\
\hline Rigo et al. ${ }^{10}$ & PT $F_{6}$ & $135 \pm 4$ & $46 \pm 13$ & $59 \pm 17$ & $88 \pm 3$ & $91 \pm 5$ & $53 \pm 7$ \\
\hline Schanler et al. ${ }^{8}$ & & 147 & 79 & 74 & 69 & 61 & 50 \\
\hline Rowe et al. ${ }^{11}$ & & 219 & 166 & 159 & 116 & 89 & 87 \\
\hline
\end{tabular}

Adapted from Koo \& Tsang $^{4}$ and Rigo et al. ${ }^{2}$ 
The calcium content supplied per $\mathrm{kg} / \mathrm{day}$ varies considerably for both supplemented breastmilk and milkbased formulas. In breastmilk, phosphorus supplementation is a way to regulate calcium retention, especially because the phosphorus content in breastmilk is low. In formulas, precipitation or the lower solubility of calcium can interfere in its absorption. ${ }^{12}$ However, as observed in Table 1, some formulas with high calcium content result in calcium retention compatible with the needs for appropriate mineralization. In preterm formulas, an appropriate $\mathrm{Ca}: \mathrm{P}$ ratio should be observed by proposing a ratio close to $2: 1$ and by evaluating the bioavailability of calcium salt and the amount of phosphorus used. ${ }^{2}$

There is no doubt that breastmilk supplementation with $\mathrm{Ca}$ and $\mathrm{P}$ increases the amount of calcium retained by the preterm infant. However, the analysis of the effect on the bones has not shown clearly evident results. The evaluation performed by Cochrane Review in $2004^{13}$ did not include randomized studies on newborns who received only calcium and phosphorus supplementation or studies that evaluated bone growth and metabolism of preterm infants. Most studies evaluate only metabolic parameters, especially the use of breastmilk supplements with multiple components.

The analysis of several studies done by Cochrane Review ${ }^{14}$ on breastmilk containing supplements and different components such as proteins, fat, carbohydrate, minerals, electrolytes and microelements, with at least two of the elements mentioned, initiated when the newborn could tolerate a predefined ingestion, showed an increase in gain weight, length and head circumference without differences in serum alkaline phosphatase levels in the short run. The results regarding bone mineralization are discrepant, since only two studies described an increase in the BMC of the radius $^{15}$ and an increase in total $B M C, 16$ while two authors 17,18 did not find differences in total BMC with the supplementation. No fractures, hypercalcemia or any other clinical manifestations were observed.

Preterm formulas compared to supplemented breastmilk provide greater longitudinal growth; however, mineralization is similar but smaller than that which is observed in utero. ${ }^{2}$

When deciding about the type of nutrition, other factors in favor of the use of supplemented breastmilk should be considered. Metabolic balance studies indicate that calcium absorption is a linear function of ingestion, with a range from 40 to $120 \mathrm{mg} / \mathrm{kg} /$ day of supplemented breastmilk or preterm formulas. ${ }^{5}$

\section{Metabolic bone disease}

It includes bone mineralization disorders found in newborn infants, ranging from hypomineralization, defined as osteopenia of prematurity, to more intense findings, characterizing rickets of prematurity. The frequency of metabolic bone disease amounts to $50 \%$ in preterm infants weighing less than $1,000 \mathrm{~g}$ and $30 \% 2$ in those weighing less than $1,500 \mathrm{~g}$.

The clinical diagnosis is established between 2 and 4 months and it is characterized by signs of rickets with posterior skull deformity, thickening of the chondrocostal junctions, enlargement of the distal portions of the radius and cubitus, enlargement of the anterior fontanelle and of frontal bosses. Rib fractures and long bone fractures occur in $10 \%$ of very low birthweight infants. Growth can also be decreased. This is characterized as rickets of prematurity; however, hypomineralization is the most frequent manifestation, which can be evidenced by the x-ray of long bones. In this phase, when radiological signs are present, reduction in bone density has already occurred, with a $40 \%$ loss of bone minerals.

The radiological diagnosis is imprecise and depends on the severity and duration of mineral deficiency. A more accurate evaluation is that in which BMC is determined by bone densitometry using double-photon or single-photon absorption equipment, when two beams of energy are used. Double-photon absorption is more reliable to measure the BMC o the whole body.

As to the biochemical analysis, serum calcium can have normal or low levels, serum phosphorus is low, and the activity of alkaline phosphatase increases as well as that of osteocalcin. Biochemical indicators, such as alkaline phosphatase, have been considered to identify preterm infants with metabolic bone disease. The increase in alkaline phosphatase, with a cutoff point corresponding to five times the reference value for normal adults, has been used in clinical practice. However, conflicting conclusions are observed in studies that relate alkaline phosphatase with BMC determined by photon absorption. Thus, some authors reported a weak association of phosphatase and serum phosphorus with BMC, while other authors such as Faerk et al. ${ }^{19}$, in 2002, did not observe any association between BMC and alkaline phosphatase or serum phosphorus in preterm infants, considering their gestational age. Nevertheless, a significant negative association between phosphatase and serum phosphorus was observed when 127 preterm infants aged less than 32 weeks of gestation were evaluated. They used dual-energy $x$-ray absorptiometry (DEXA) and scanning of the whole body, a method that is more accurate than those used by other researchers, and concluded that routine measurements of alkaline phosphatase and serum phosphorus are not useful in predicting the outcome of bone mineralization in preterm infants.

Although one may consider that calcium and phosphorus deficiency is the main cause of bone diseases in preterm infants, it has been shown that the increase in bone resorption ${ }^{20}$ contributes to bone diseases in preterm infants, from the moment in which resorption markers, such as hydroxyproline obtained from the collagen turnover, are detected in the blood of preterm infants. In addition, even when formulas with high calcium and high phosphorus concentrations are used, the BMC of preterm infants is lower than reference values. According to Miller, ${ }^{20}$ in 2003, the variables that could explain the differences in bone resorption of preterm infants are biomechanical factors, whereby pressure on the bones stimulates osteoblasts that produce bone and osteoclasts that reabsorb the bone, resulting in bone remodeling. This pressure results from 
movements performed by the fetuses against the uterine wall, in contrast to what occurs with preterm infants outside the uterus. Miller ${ }^{20}$ suggests that the amount of minerals to be supplied in the postnatal period be reassessed, since they may not reflect intrauterine requirements.

Other factors observed in extremely preterm infants that influence bone growth and mineralization are longterm parenteral nutrition and the use of drugs with a hypercalciuric effect, such furosemide and methylxanthines.

\section{Effects of nutrition after birth on short and long- term bone growth and mineralization}

At hospital discharge, extremely low birthweight infants often show growth retardation ${ }^{21}$ and low bone mineralization as a result of their difficulty in receiving proper nutrition. Epidemiological studies suggest that intrauterine and postnatal growth retardation may predispose to adult diseases. However, late skeletal consequences still need to be determined.

Short-term effects of low calcium and phosphorus intake, resulting in a low BMC at hospital discharge, have been reported by several authors. According to Rigo et al., ${ }^{2}$ in 2000, mineralization compensation may occur around the 12th postnatal week. Nevertheless, long-term studies are scarce and difficult to evaluate because of different nutritional protocols for preterm infants. There are signs that BMC deficit disappears in childhood, especially if they are corrected based on anthropometric data. Sixty-four preterm infants with less than 32 weeks of gestational age, among whom 30 were extremely preterm infants (gestational age less than 28 weeks), received fortified breastmilk during their hospital stay. After hospital discharge, they received breastmilk or usual formula. When they reached between 5.0 and $7.0 \mathrm{~kg}$, BMC of the spine was assessed by dual-energy absorptiometry. At discharge, $70 \%$ of them had developed growth deficit. With exclusive breastfeeding, the linear weight gain was maintained, but with increased risk (OR $7.0 ; 95 \%$ CI 1.2-41.7) of having low BMC values. Low serum phosphorus at six postnatal weeks was considered a risk factor for hypomineralization. ${ }^{22}$ The authors concluded that the possibility of fortification of human milk after hospital discharge should be assessed. ${ }^{22}$ In another study, ${ }^{23}$ two groups of preterm infants who received human milk without and with calcium supplementation ( $108 \mathrm{mg} / \mathrm{kg} /$ day) and phosphorus supplementation ( $53 \mathrm{mg} / \mathrm{kg} /$ day) until they reached $2,000 \mathrm{~g}$ were examined at three months. BMC was $36 \%$ greater than in the group that received supplementation. However, when examined at 9 and 11 years, mineralization was the same in both groups and appropriate for age. ${ }^{23}$

Fewtrell et al.24, in 1999, assessed children at 8-12 years who had been born prematurely and fed human milk, preterm or full-term formulas, and concluded that preterm infants were smaller than full-term newborns at 8-12 years and that BMC, albeit lower, was appropriate for age, indicating that differences in mineral intake did not affect bone mass in infants born prematurely and examined at 812 years. These authors found that it is necessary to enhance growth in order to improve bone mass.
Most studies on the effects of hypomineralization at previous stages of life concern the long-term evaluation of cohorts analyzed in previous years and that, consequently, are flawed, since they were not designed for this purpose. In general, the patient population is small and these studies sometimes compare preterm infants who received diets poor in minerals at the time. Neither do these studies deal with very low birthweight or extremely low birthweight infants, who are more susceptible to osteopenia. In addition, there is no reference to nutrition after hospital discharge. Therefore, randomized controlled trials involving extremely preterm infants can point out the late consequences of hypomineralization in the postnatal period.

\section{Nutrition of preterm infants after hospital discharge and the effects on bone mineralization}

At hospital discharge, extremely low birthweight infants have remarkable growth retardation and complications that may affect nutrient intake and cause protein-energy malnutrition after discharge, in addition to poor mineralization. Although most of these preterm infants have a catch up growth after hospital discharge, growth is still smaller in comparison with that of full-term newborns.

Currently, some data suggest that preterm formulas fortified with nutrients after hospital discharge can significantly increase weight, length, head circumference and bone mineralization, compared to standard formulas for full-term infants. ${ }^{25,26}$ The effects on growth are more evident in the first months and in male newborns. ${ }^{26}$

Two types of fortified formulas have been used: especially prepared formulas known as post-discharge formulas or preterm formulas similar to the ones used during hospitalization. They are also used as breastmilk supplements. Post-discharge formulas have a higher protein and energy content, supplements of $\mathrm{Ca}, \mathrm{P}$, zinc and microelements. ${ }^{27}$ The preliminary studies by Lucas et al. ${ }^{28,29}$ in which, after hospital discharge, they fed preterm infants weighing less than $1,850 \mathrm{~g}$ a formula containing proteins and minerals, showed greater growth and bone mineralization than full-term formulas, and hence, they created the concept of post-discharge formula. Griffin ${ }^{30}$ reviews several ways of nutritional supplementation for preterm infants after hospital discharge. Benefits are not so evident and are even controversial sometimes, and therefore, new assessments that can provide more definite conclusions are necessary. There is a study in which preterm infants, after receiving post-discharge formula up to the ninth month of postnatal age, had a greater BMC than formula-fed full-term newborns. Also, these patients had heavier weight, greater length and larger head circumference. Other authors, using similar formulas for the same period of time, described an increase in weight and length at nine months, but at 18 months, only differences in length persisted. Another study in which post-discharge formula was given up to 12 months of corrected age, preterm infants began to ingest a lower volume, equalizing the amount of energy to that obtained from the full-term formula. Weight differences occurred at earlier stages. 
There is a paucity of studies indicating whether the formulas used for preterm infants should continue to be used after hospital discharge. However, these formulas, containing different components often have high amounts of vitamins and minerals, calculated so as to allow for growth and increase in mineral level similar to that observed in utero, and that do not necessarily correspond to postnatal requirements. There was an increase in plasma zinc concentration and an improvement in length and head circumference only in boys. Another study did not show any differences in serum concentrations of calcium, phosphorus and alkaline phosphatase.

In a review article, Fewtrell ${ }^{27}$ considers that postdischarge or preterm formulas imply better growth, and that they should be preferred to full-term formulas after the discharge of preterm infants.

Studies about supplementation of breastmilk in the post-discharge period are concerned with regular supplements, with increase in the content of proteins or vitamins or minerals, or even the association of these components. Supplementation of breastmilk with calcium, phosphorus and vitamin D, after hospital discharge, reduced the incidence of hypophosphatemia. Formulas supplemented with calcium and phosphorus used for a short period of time after hospital discharge, for 8 weeks, showed an increase in BMC after this period, which may not persist in the long run. 30

\section{Other minerals: trace elements or microelements}

Important microelements or trace elements to human nutrition include zinc, copper, selenium, chrome, molybdenum, manganese, iodine and iron. Although they quantitatively represent a small fraction of the total mineral content of the human body, they play a key role in several metabolic pathways. Preterm infants may have deficiencies, even if clinical manifestations are absent, due to low stores at birth, since mineralization occurs in the last trimester of gestation. Very little is known about the metabolism of these trace elements in the nutrition of extremely preterm newborns, but there is a lot of information about preterm infants or very low birthweight infants.

\section{Selenium}

This element has taken on added importance in all age groups, since it is a constituent part of selenoenzymes with different functions, including glutathione peroxidase, which is an antioxidant enzyme that acts on cell membranes. These enzymes prevent the formation of free radicals, reducing lipid peroxides and hydrogen peroxide, protecting the body against oxidative insult. Selenium also plays a role in immunocompetence. Neutrophils and macrophages of selenium-deficient animals have a low concentration of glutathione peroxidase, which may affect their antimicrobial properties. ${ }^{5,31}$

Extremely low birthweight infants are at risk for conditions whose pathophysiology includes free radical damage, as observed in bronchopulmonary dysplasia, retinopathy of prematurity, peri-intraventricular hemorrhage and necrotizing enterocolitis. Also due to the frequency at which they receive oxygen, they are susceptible to oxidative stress and production of reactive oxygen. Although there are signs that selenium deficiency participates in diseases of prematurity, there is a dearth of data that could corroborate its actual importance. In a review article, Klein ${ }^{5}$ mentions studies that relate selenium deficiency to diseases of prematurity. Thus, eight preterm newborns who developed respiratory distress syndrome and who did not receive oral or parenteral selenium revealed a quick decrease in serum levels two weeks after birth. Also, preterm infants with chronic lung disease and who did not receive selenium supplementation showed a remarkable decrease in selenium concentrations. In New Zealand, a country where the soil and foods are poor in selenium, low selenium levels in 79 very low birthweight preterm infants were associated with the increase in morbidity and plasma concentrations were significantly lower in oxygen-dependent infants at 28 postnatal days. The effects of low selenium concentrations in newborns have not been established yet. They have been suggested as a risk factor for chronic lung disease and retinopathy of prematurity in preterm infants, and there has been uncertainty about whether selenium supplementation reduces the risk for these diseases.

\section{Selenium in the fetus and newborn}

The control mechanisms of the mother-to-fetal transfer of selenium have not been clearly defined yet. Transplacental transfer is limited, so selenium concentration is relatively low in the fetus, corresponding in umbilical cord blood to approximately $65 \%$ of the maternal serum level. We observed selenium umbilical cord blood concentrations without significant differences in very low birthweight infants compared to full-term newborns, either appropriate-for-gestational-age or smallfor-gestational-age, corresponding to approximately $80 \%$ of maternal levels. ${ }^{32}$ However, Makhoul et al. ${ }^{33}$ analyzed selenium in the umbilical cord blood of newborns with 24 to 42 weeks of gestational age and found a significant association with gestational age and a remarkable increase in selenium concentrations after 36 weeks.

No data exist on the fetal concentration of selenium, but its mineralization is estimated at $1 \mu \mathrm{g} / \mathrm{kg} /$ day. Selenium is stored in fetal liver between the 20th and 40th week, and may be held responsible for low levels found in preterm newborns. In the literature, some studies analyze selenium concentrations in very low birthweight infants, showing that plasma concentrations of selenium and glutathione peroxidase are correlated with birthweight and that, in very low birthweight infants, the activity of glutathione peroxidase corresponds to one third of that which is observed in fullterm newborns. ${ }^{34}$

In preterm infants with respiratory disorders, submitted to parenteral nutrition without selenium supplementation in the first 14 days, there was a relevant decrease in selenium levels in the first days of life. Concentrations increased when 
a selenium-containing formula was used, except for one newborn who developed bronchopulmonary dysplasia. ${ }^{35}$ Other authors have shown a progressive reduction in the activity of glutathione peroxidase and selenium in erythrocytes of preterm infants in the second week of life when they received a standard milk-based formula.

In a study conducted at the neonatal unit of the School of Medicine of Botucatu (UNESP), we assessed serum selenium levels in three groups of newborns: very low birthweight infants, appropriate-for-gestational age fullterm infants, and small-for-gestational age full-term infants, from birth to 30 days of chronological age. These newborns did not receive selenium supplementation via parenteral or enteral nutrition. Umbilical cord blood selenium levels were lower than maternal levels. The only differences were the significantly lower levels in very low birthweight infants on the 3rd, 15th and 30th days in relation to appropriate-forgestational-age and small-for-gestational-age full-term infants, as well as a progressive reduction in selenium levels in the first month of life only in preterm infants. ${ }^{32}$ These results allow us to conclude about the necessity to supplement the nutrition of very low birthweight infants with selenium given parenterally and enterally. This practice has been mentioned by Darlow et al. ${ }^{36}$ in a multicenter, randomized, double-blind study that included 534 very low birthweight assessed at 28 days and 36 weeks of postconceptional age. The treated group received $7 \mu \mathrm{g} / \mathrm{kg} /$ day parenterally and $5 \mu \mathrm{g} / \mathrm{kg} /$ day supplemented with breastmilk or formula. The authors noted that glutathione peroxidase and selenium levels were significantly lower in the group that did not receive supplementation at 28 days and 36 weeks of corrected age. These observations were also confirmed by most studies on selenium with preterm infants. However, whether supplementation is beneficial in the prevention of diseases in newborn infants still has to be evaluated. In a meta-analysis carried out by Darlow \& Austin, 37 three studies met the requirements and included 297 newborn infants who received selenium supplementation and 290 controls. Two studies included extremely preterm infants. Among complications, they assessed the need for oxygen at 28 days, episodes of sepsis, in-hospital death, chronic lung disease (need of oxygen at 28 days and 36 weeks of postconceptional age) and retinopathy. As a conclusion, they reported that selenium supplementation reduced one or more episodes of late-onset sepsis, but no significance was found for the other diseases or complications.

The amount of selenium used for supplementation in preterm infants is variable. In the United States, $2 \mu \mathrm{g} / \mathrm{kg} / \mathrm{day}$ given parenterally is recommended. Nevertheless, a metaanalysis suggested $3 \mu \mathrm{g} / \mathrm{kg} /$ day in order to maintain concentrations at the umbilical cord blood levels. ${ }^{37}$ To increase the concentrations above umbilical cord blood levels and bring them closer to those of breastfed full-term infants, $5-7 \mu \mathrm{g} / \mathrm{kg} /$ day is recommended. Other factors that influence selenium levels are the pathways used, biochemical forms and the content of selenium in the formulas used. 37

Experts gathered in a panel organized by the Food and Drug Administration and American Society for Nutritional Sciences ${ }^{5}$ recommend a minimum selenium concentration of $1.8 \mu \mathrm{g} / 100 \mathrm{kcal}$ and a maximum concentration of $5.0 \mu \mathrm{g} / 100 \mathrm{kcal}$ in preterm formulas.

\section{Zinc}

Zinc is perhaps the most widely studied microelement in infant feeding as it is important to growth, cell differentiation, and to the metabolism of proteins, carbohydrates and lipids. It plays a role in hormone structure and in genetic transcription factors. ${ }^{38}$ Signs of subclinical zinc deficiency may occur and are similar to the deficiency of other dietary components, due to its large scope of action, if the preterm infant remains for a long time on parenteral nutrition without zinc supplementation. More characteristic manifestations develop after 3 months, including weight loss, failure to thrive, periorificial dermatitis, glossitis, and enhanced susceptibility to infections. ${ }^{31}$ Some factors contribute to the development of deficiencies, such as preterm birth, depriving the newborn infant of fetal mineralization of zinc, which, similarly to other micronutrients, accumulates in the third trimester of gestation. Preterm infants also have an immature gastrointestinal tract, which results in negative zinc balance, with zinc excretion by the intestinal tract. Studies involving isotope-labeled compounds show that preterm infants are able to take up exogenous zinc, increasing its absorption and reducing its excretion, but the patterns of this behavior in extremely preterm infants are still unknown. Preterm infants can take up $25 \%$ to $40 \%$ of dietary zinc. ${ }^{5}$ In addition to lower enteral uptake and lower hepatic reserve, compared to other microelements, formula components, such as iron, can affect zinc bioavailability in the presence of a high Fe: $\mathrm{Zn}$ ratio. Interference of iron with zinc is observed in adults and is arguable in preterm infants. However, Klein ${ }^{5}$ states that a $2: 1 \mathrm{Fe}: \mathrm{Zn}$ ratio should be used in the formulas, with a maximum of $3.0 \mathrm{mg}$ of zinc for $1.5 \mathrm{mg}$ of iron per $100 \mathrm{kcal}$.

Zinc bioavailability in breastmilk is greater than in cow's milk; therefore, zinc concentration should be high in the supplied formulas. The difference in bioavailability is due to the strong binding of zinc to casein. ${ }^{5}$ It has been shown that $60 \%$ of zinc found in the milk for preterm infants is absorbed compared to $36 \%$ of zinc found in fortified breastmilk and $14 \%$ found in preterm formulas. Zinc concentration in breastmilk quickly decreases after the colostrum, when levels are at their highest. 38

Growth is the major factor used to determine zinc requirements of preterm infants. Calculations of zinc requirements for preterm infants between 24 and 28 weeks of gestational age indicate a requirement of $600 \mu \mathrm{g} /$ day for the formation of new tissues (except zinc for storage), necessary to provide growth similar to the one achieved by a 40 -weeker.The release of hepatic zinc by a newborn weighing $1,000 \mathrm{~g}$ is on average $150 \mu \mathrm{g}$ /day. Extremely preterm infants have fewer reserves and depletion occurs earlier, around 32-36 weeks. The calculation of dietary zinc in order for optimal growth to occur is equivalent to $500 \mu \mathrm{g} / \mathrm{kg} / \mathrm{day}$, with approximately $1,000 \mathrm{~g}$ of birthweight and 27 weeks of gestational age; $400 \mu \mathrm{g} / \mathrm{kg} /$ day for newborn infants between 1,500 and $2,000 \mathrm{~g}$ (30-32 weeks) 
and $200-300 \mu \mathrm{g} / \mathrm{kg} /$ day for those weighing between 2,500 and $3,500 \mathrm{~g}$ (35-40 weeks). ${ }^{5}$

Given the low absorption of zinc from formulas, an expert panel in $2002^{5}$ recommended that the minimum and maximum concentrations in preterm formulas be $1.1 \mathrm{mg} /$ $100 \mathrm{kcal}$ and $1.5 \mathrm{mg} / 100 \mathrm{kcal}$. In the initial period, authors recommended $500-800 \mu \mathrm{g} / \mathrm{kg} /$ day, increasing to $1 \mathrm{mg} / \mathrm{kg} /$ day when growth is established. ${ }^{38}$ In stable preterm infants, $400 \mu \mathrm{g} / \mathrm{kg} /$ day given parenterally is recommended, while for the transition period (up to 2 weeks), the recommendation is of $50 \mu \mathrm{g} / \mathrm{kg} /$ day. ${ }^{38}$

\section{Other minerals}

In the literature, the concern with iron supplementation is far more related to the morbidity of preterm infants than to nutritional aspects. Studies focus on the prevention and treatment of anemia of prematurity.

Minerals such as copper, iodine, manganese and molybdenum have been investigated as to the feeding of preterm infants in order to develop special preterm formulas and parenteral nutrition solutions. There are few references about the requirements of extremely preterm infants and, therefore, we should consider the publications about low birthweight full-term or preterm infants. Clinical manifestations caused by deficiency in the neonatal period are not described, that is why their characteristics are underreported in daily practice.

Among these elements, special attention should be paid to copper, an important element in the constitution of enzymes such as superoxide dismutase, which protects the cell membranes against oxidative damage. This element is stored in fetal liver, being bound to metallothionein in greater amounts than those found in the liver of adult individuals. Nevertheless, transport from the liver to the tissues is performed by ceruloplasmin, whose levels are low in newborns, and whose production starts 6 or 12 weeks after birth. 5,31,38 Deficiency is rare in the neonatal period, but when present, it causes hypochromic anemia resistant to iron supplementation, neutropenia, osteoporosis, skin manifestations and difficulty in gaining weight. There is a paucity of data about copper requirements in preterm infants. When fed breastmilk, they receive enough quantities. On long-term parenteral nutrition, it is recommendable to use $20 \mu \mathrm{g} / \mathrm{kg} /$ day and not use it in the presence of cholestasis, because it is excreted by the bile. ${ }^{38}$

We hope that further research is conducted about the role of minerals in preterm nutrition, so that formulas can be more balanced in terms of mineral contents and more appropriate for the requirements of extremely preterm infants, who usually are submitted to parenteral nutrition for a longer period.

\section{References}

1. Greer FR, Tsang RG. Calcium, phosphorus, magnesium and vitamin $D$ requirements for the preterm infant. In: Tsang RC, editor. Vitamin and minerals requirements in preterm infants. New York: Marcel Deker; 1985. p. 99-136.
2. Rigo J, De Curtis M, Pieltain C, Picaud J, Salle BL, Santerre J. Bone mineral metabolism in the micropremie. Clin Perinatol. 2000;27:147-70.

3. Venkataraman PS, Tsang RC, Chen IW, Sperling MA. Pathogenesis of early neonatal hypocalcaemia: studies of serum calcitonin, gastrin, and plasma glucagon. J Pediatr. 1987;110:559-603.

4. Koo WW, Tsang RC. Calcium, magnesium, phosphorus and vitamin D. In: Tsang RC, Lucas A, Uauy R, Zlotkin S, editors. Nutrition needs of the preterm infant. Scientific basis and practical guidelines. Baltimore: Williams \& Wilkins; 1993. p. 135-55.

5. Klein $\mathrm{C}$. Nutrient requirements for preterm-infant formulas: 10. Minerals: calcium and phosphorus. J Nutr. 2002;132(6 Suppl 1):S1395-577.

6. Salle B, Senterre J, Putet G, Rigo J. Effects of calcium and phosphorus supplementation on calcium retention and fat absorption in preterm infants fed pooled human milk. J Pediatr Gastroenterol Nutr. 1986;5:638-42.

7. Ehrenkranz RA, Gettner PA, Nelli CM. Nutrient balance studies in premature infants fed premature formula or fortified human milk. J Pediatr Gastroenterol Nutr. 1989;8:58-67.

8. Schanler RJ, Abrams AS, Garza C. Bioavailability of calcium and phosphorus in human milk fortifiers and formula for very low birth weight infants. J Pediatr. 1988;113:95-100.

9. Rigo J, Salle BL, Picaud JC, Putet G, Senterre J. Nutritional evaluation of protein hydrolysate formulas. Eur J Clin Nutr. 1995;49 Suppl 1:S26-38.

10. Rigo J, Picaud JC, Lapellone A, Salle LB, Senterre, J. Metabolic balance and plasma AA concentrations in VLBW infants fed a new acidic whey hydrolysate preterm formula. J Pediatr Gastroenterol Nutr. 1997;24:491A.

11. Rowe JC, Goez CA, Carey DE, Horak E. Achievement of in uterus retention of calcium and phosphorus accompanied by high calcium excretion in very low birth weight infants fed a fortified formula. J Pediatr. 1987;110:581-5.

12. Salle BL, Senterre J, Putet G. Calcium, phosphorus, magnesium and vitamin $D$ requirements in premature infants. In: Salle $B L$, Swyer PR, editors. Nutrition of the low birth weight infant. Nestlé Nutrition Workshop Series, vol. 32. New York: Raven Press; 1993. p. 125-34.

13. Kuschel CA, Harding JE. Calcium and phosphorus supplementation of human milk for preterm infants. Cochrane Database Syst Rev. 2001;(4):CD003310.

14. Kuschel CA, Harding JE. Multicomponent fortified human milk for promoting growth in preterm infants. Cochrane Database Syst Rev. 2004;(1):CD000343.

15. Mondanlou HD, Lim MO, Hansen GW, Sickles V. Growth, biochemical status and mineral metabolism in very-low birth weight infants receiving fortified preterm human milk. J Pediatr Gastroenterol Nutr. 1986;5:762-7.

16. Pettifor JM, Rajah R, Venter A, Moodley GP, Opperman L, Cavaleros $\mathrm{M}$, et al. Bone mineralization and mineral homeostasis in very-low-birth-weight infants fed either human milk or fortified human milk. J Pediatr Gastroenterol Nutr. 1989;8: 217-24.

17. Wauben IP, Atkinson SA, Grad TL, Shah JK, Paes B. Moderate nutrient supplementation of mother's milk for preterm infants supports adequate bone mass and short-term growth: a randomized, controlled trial. Am J Clin Nutr. 1998;67:465-72.

18. Faerk J, Petersen B, Petersen S, Michaelsen KF. Diet and bone mineral content at term in premature infants. Pediatr Res. 2000;47:148-56.

19. Faerk J, Petersen B, Petersen S, Michaelsen KF. Bone mineralisation in premature infants cannot be predicted from serum alkaline phosphatase or serum phosphate. Arch Dis Child Fetal Neonatal Ed. 2002;87:133-6.

20. Miller ME. The bone disease of preterm birth: a biomechanical perspective. Pediatr Res. 2003;53:10-5.

21. Berry MA, Conrad H, Usher RH. Growth of very premature infants fed hyperalimenation and calcium-supplemented formula. Pediatrics. 1997; 100:647-53.

22. Kurl S, Heinonen K, Lausimies E. Pre-and post-discharge feeding of very preterm infants: impact on growth and bone mineralization. Clin Physiol Func Imagin. 2003;23:182-9.

23. Backström MC, Mäki R, Kuusela AL, Sievänin $H$, Koivisto A-M, Koshinen $M$, et al. The long-term effect of early mineral, vitamin $\mathrm{D}$, and breast milk intake on bone mineral status in 9-11-yearold children born prematurely. J Pediatr Gastroenterol Nutr. 1999;29:575-82. 
24. Fewtrell MS, Prentice A, Jones SC, Bishop NJ, Stirling D, Buffenstein $\mathrm{R}$, et al. Bone mineralization and turnover in preterm infants 8-12 years of age: the effect of early diet. J Bone Miner Res. 1999;14:810-20.

25. Carver JD, Wer PYK, Hall RT, Ziegler EE, Sosa R, Jacobs J, et al. Growth of preterm infants fed nutrient enriched or term formula after hospital discharge. Pediatrics. 2001;107:683-9.

26. Carver JD. Advances in nutritional modifications of infant formulas. Am J Clin Nutr. 2003;77:S1550-4.

27. Fewtrel MS. Growth and nutrition after discharge. Semin Neonatol. 2003;8:169-70.

28. Lucas A, King F, Bishop NB. Postdischarge formula consumption in infants born preterm. Arch Dis Child. 1992;67:691-2.

29. Lucas A, Fewtrell MS, Morley R, Singhal A, Abbot RA, Isaaco E, et al. Randomized trial of nutrient-enriched formula versus standard formula for postdischarge preterm infants. Pediatrics. 2001;108:703-11.

30. Griffin IJ. Postdischarge nutrition for high risk neonate. Clin Perinatol. 2002;29:327-44.

31. Agget PJ. Trace elements of the micropremie. Clin Perinatol. 2000;27:119-29.

32. Daher S, Trindade CE, Rezende C, Miranda A, Crossi R. Blood selenium levels of very low birth weight infants during the first month of life. Pediatr Res. 2001;49:297A.

33. Makhoul IR, Sammour RN, Diamond E, Shohat I, Tamir A, Shamir R. Selenium concentrations in maternal and umbilical cord blood at 24-42 weeks of gestation: basis for optimization of selenium supplementation to premature infants. Clin Nutr. 2004;23:373-81.
34. Locktich G, Jacobson B, Quigley G, Dison P, Pendray M. Selenium deficiency in low birth weight neonates: are unrecognized problem. J Pediatr. 1989;114:865-70.

35. Amin S, Chen SY, Collipp PJ, Castro MN, Maddalaiah VT, Klein SW. Selenium in premature infants. Nutr Metab. 1980;24:331-40.

36. Darlow BA, Winterbourn CC, Irider TE, Graham PJ, Harding JE, Weston PJ. The effect of selenium supplementation on outcome in very low birth weight infants: a randomized controlled trial. The New Zeland Neonatal Study Group. J Pediatr. 2000;136: 473-80.

37. Darlow BA, Austin NC. Selenium supplementation to prevent short-term morbidity in preterm neonates. Cochrane Database Syst Rev. 2003;(4):CD003312.

38. Reifen RM, Zlotkin S. Microminerals. In: Tsang RC, Lucas A, Uauy $R$, Zlotkni $S$, editors. Nutritional needs of the preterm

Correspondence:

Cleide Enoir Petean Trindade

Rodovia Domingos Sartori, km 01

CEP: 18607-741 - Botucatu, SP

Brazil

Phone: + 55 (14) $3811.6274 / 3882.1472$

Fax: + 55 (14) 3811.6274

E-mail: pediatri@fmb.unes.br 